\title{
Analyse d'ouvrage par Marie Ghis Malfilatre
}

Pascal Ughetto (2018). Les nouvelles sociologies du travail. Introduction à la sociologie de l'activité

Marie Ghis Malfilatre

\section{OpenEdition}

Journals

Édition électronique

URL : http://journals.openedition.org/activites/6273

DOI : 10.4000 /activites.6273

ISSN : $1765-2723$

Éditeur

ARPACT - Association Recherches et Pratiques sur les ACTivités

Référence électronique

Marie Ghis Malfilatre, «Analyse d'ouvrage par Marie Ghis Malfilatre », Activités [En ligne], 18-1 | 2021, mis en ligne le 15 avril 2021, consulté le 17 avril 2021. URL : http://journals.openedition.org/activites/ 6273 ; DOI : https://doi.org/10.4000/activites.6273

Ce document a été généré automatiquement le 17 avril 2021

\section{(c) (i) (3) $\Theta$}

Activités est mis à disposition selon les termes de la licence Creative Commons Attribution - Pas d'Utilisation Commerciale - Pas de Modification 4.0 International. 


\section{Analyse d'ouvrage par Marie Ghis Malfilatre}

Pascal Ughetto (2018). Les nouvelles sociologies du travail. Introduction à la sociologie de l'activité

\section{Marie Ghis Malfilatre}

\section{RÉFÉRENCE}

Pascal Ughetto (2018). Les nouvelles sociologies du travail. Introduction à la sociologie de l'activité. Louvain-la-Neuve : De Boeck, 152 p.

1 L'ouvrage de Pascal Ughetto a pour ambition de rendre compte des renouvellements introduits dans la sociologie du travail en France à partir des années 1990 par un large ensemble de recherches regroupées ici sous le label de "sociologie de l'activité ». Destiné à un public d'étudiants, de praticiens, mais aussi de sociologues intéressés par d'autres domaines, l'ouvrage entend mettre à disposition ces travaux et leurs méthodes pour «aider à s'emparer de cette ressource théorique qui vient s'ajouter aux possibilités diverses d'analyse sociologique du travail» (p.15). Organisé en six chapitres, ce court ouvrage entend ainsi présenter de manière pédagogique un ensemble de recherches, en préciser les origines, mais aussi engager une discussion autour de leurs apports et de leurs limites.

L'introduction s'ouvre sur le contexte qui a vu émerger ces «nouvelles sociologies du travail» dans un moment de reconfiguration $d u$ paysage intellectuel français caractérisé à la fois par le recul du marxisme et un «tournant pragmatique » dans les sciences sociales à la fin des années 1980 (p.12). Les problématiques classiques de la sociologie du travail, principalement centrées sur la figure de l'ouvrier et pensées à travers le prisme salarial, peuvent se répartir en quatre pôles principaux selon Pascal Ughetto. D'un côté se trouvent les travaux portant sur les enjeux liés à la division et à la rationalisation du travail ou sur les problématiques de qualifications et de métiers et, d'un autre côté, les recherches sur l'évolution des formes d'emploi ou sur les relations 
professionnelles (pp. 8-12). Par contraste, les «nouvelles sociologies du travail», aussi plurielles soient-elles, partagent un intérêt commun pour l'analyse du « travail en train de se faire » ce qui, au plan international, relève d'un mouvement nommé le " practice turn» (p.12). Ce mouvement rassemble en France des auteurs et propositions théoriques aussi diverses que l'économie des conventions de Laurent Thévenot et Luc Boltanski, la sociologie des sciences et des techniques telle que pratiquée par Michel Callon et Bruno Latour ou encore les travaux de Nicolas Dodier sur les relations que les opérateurs entretiennent avec les machines.

3 L'auteur défend que ces différents travaux ont en commun de voir le travail comme de " l'activité et des actions, là où la sociologie préférait habituellement le définir à partir des rapports sociaux découlant de la relation de subordination propre au salariat " (p.15). Dans cette perspective, le regard de l'enquêteur - qu'il soit sociologue, ergonome ou psychologue du travail - se porte avant tout sur le travail en train de se faire et ce qui l'empêche, l'objectif étant de décrire l'intelligence que les travailleurs mettent en œuvre « d'abord dans le combat avec les épreuves du travail, plus que dans la résistance à l'employeur » (p. 16). L'ouvrage vise ainsi à rendre compte des apports et des renouvellements que ces recherches ont introduits dans la pratique des sciences sociales et la compréhension des enjeux relevant du travail, mais il entend également engager un dialogue interdisciplinaire et avec les tenants des perspectives plus classiques de la sociologie du travail. Dans cette perspective, l'auteur souligne notamment que si la sociologie de l'activité introduit une rupture dans les manières de conduire les enquêtes et de problématiser les enjeux liés au travail, celles-ci ne sont pas toujours radicalement nouvelles. Il reste par ailleurs à cette approche de mieux prendre en compte « la relation de subordination » ou « les pouvoirs économiques qui permettent à certains acteurs de commander le travail d'autrui et de s'approprier la valeur qu'il contribue à créer » (p. 17).

4 Le premier chapitre revient sur les racines parfois anciennes de ces «nouvelles sociologies du travail». Celles-ci plongent dans quatre grands courants, suivant le découpage emprunté à Christian Licoppe : la cognition distribuée et l'action située, la théorie de l'activité, les recherches d'inspiration ethno-méthodologique et la sociologie des sciences et des techniques. Les recherches se réclamant de l'action située viennent au départ des États-Unis. Des auteurs vont montrer que les opérations cognitives ne sont pas le seul fait de la volonté humaine, mais qu'elles font système avec leurs environnements, y compris avec les objets qui les constituent (p. 20). Les travaux d'Edwin Hutchins sur les pilotes d'avion ou sur les équipages de navires montrent par exemple comment les décisions de haute importance concernant la conduite dans ces deux contextes s'appuient sur des repères, des indices, qui cristallisent des pratiques passées, des savoirs accumulés et qui épargnent ainsi une prenante activité de calcul permanent (p.21). Cette approche trouve des prolongements en France dans les travaux de Nicolas Dodier et sa notion «d'appuis conventionnels» ou dans ceux de Francis Chateauraynaud et son concept de "prises» (p. 21). Dans un autre style, la théorie de l'activité renvoie à la psychologie russe de l'entre-deux-guerres et à des auteurs comme Vygotski et Leontiev. Elle s'incarne en France dans la «clinique de l'activité » portée par Yves Clot au Conservatoire national des arts et métiers (CNAM). Trois propositions principales fondent cette approche : la construction du sujet se fait dans l'activité, cette activité est sociale et doit beaucoup au collectif dans lequel le sujet est en relation et, enfin, l'activité tout comme le rapport à l'objet du travail passent par des « artefacts », qui exercent la « fonction cruciale d'instrument de l'activité » (p. 22). 
L'ethnométhodologie vient pour sa part des travaux développés initialement aux ÉtatsUnis dans les années 1950 par le sociologue Harold Garkinkel, ancien élève de Talcott Parsons. Dans cette perspective, l'accomplissement pratique des opérations qui tissent notre vie quotidienne relève de « coordinations avec autrui et, plus généralement, avec notre environnement» (p. 24). Ces coordinations quotidiennes reposent sur l'assimilation d'" ethno-méthodes ", c'est-à-dire "des manières d'effectuer l'action reconnues compétentes dans une société et, par conséquent, attendues par autrui » (p. 22). La quatrième source d'inspiration de ces «nouvelles sociologies du travail» vient de la sociologie des sciences. À partir des années 1980, des auteurs comme Madeleine Akrich, Michel Callon et Bruno Latour s'intéressent en France au travail des scientifiques et développent la théorie de «l'acteur réseau », notamment déployée par Michel Callon à l'occasion de son enquête sur l'implantation réussie d'une variété de coquilles Saint-Jacques en baie de Saint-Brieuc (p. 25).

5 Après ce passage en revue des principales filiations théoriques de ces «nouvelles sociologies du travail ", l'auteur souligne deux points communs à ces différents courants. D'un point de vue méthodologique d'abord, la plupart de ces approches placent une exigence importante dans la collecte de matériaux par le chercheur avec une préférence marquée pour l'enquête ethnographique, la constitution de corpus de données d'observations, la capture des détails, l'analyse conversationnelle. D'un point de vue théorique ensuite, ces différents courants partagent le refus de plaquer sur les scènes observées des interprétations préexistantes. Elles veillent également à ne pas analyser le sens des situations indépendamment des significations que les acteurs impliqués leur donnent. Par ailleurs, "les postures de recherche défendues, dans l'analyse du travail comme sur d'autres sujets, s'opposent à celles - fonctionnalistes aux États unis, s'inspirant de P. Bourdieu en France, etc. - qui inscrivent les acteurs dans des ensembles structurés ou des champs, des touts qui décideraient largement des identités, des positions, des intérêts et donc du sens des actions des protagonistes » (p. 31).

6 Le second chapitre se concentre sur les travaux portant sur les relations de service. À partir de la fin des années 1980, le primat donné à l'analyse du travail ouvrier dans l'industrie est interrogé. Les services et la relation de service apparaissent alors comme un domaine de recherches particulièrement riche. En France, le rôle d'Isaac Joseph est décisif dans ce mouvement. En réponse à une commande de la RATP qui souhaite mieux comprendre les mises en inaptitude de ses agents, Isaac Joseph entreprend une enquête sur les conducteurs de bus en combinant des observations directes et des enregistrements vidéos. Il cherche à mieux comprendre ce qui, dans le métier, peut être perçu comme des situations présentant un risque ainsi que les compétences techniques et relationnelles - que les agents déploient pour y faire face ou les prévenir. Isaac Joseph montre bien que l'improvisation est monnaie courante dans le travail des agents de la RATP et que ces derniers abordent les situations présentant des risques en mobilisant des "cadres» et des «scripts » (p. 38). Par ailleurs, au moment où les grandes entreprises publiques sont l'objet de politiques visant à «moderniser " leur organisation, notamment en renforçant chez les agents des postures supposées leur faire défaut, comme celles relevant du souci de rendre un réel service aux usagers, le travail d'Isaac Joseph permet de mieux comprendre les relations ordinaires entre agents et usagers et les différentes compétences sociales dont doivent faire preuve les agents - compétences "technique», "civique», "contractuelle», mais aussi « réparatrice » - pour mener à bien leur mission (p. 39). Il éclaire toute la complexité 
du travail de la relation de service, qui se révèle infiniment plus dense que ce que les fiches de postes peuvent indiquer, la gestion de l'interaction relevant d'un "art " (p. 47). Dans le prolongement de ces travaux, une véritable "sociologie du guichet» voit le jour en France avec les travaux de Jean-Marc Weller sur la Sécurité sociale et la revisite d'une problématique toute wébérienne du travail bureaucratique. Parmi les influences qui nourrissent ce mouvement se trouvent des sociologues de l'école de Chicago, mais également, dans un autre registre, le travail du politiste Michael Lipsky sur les «street-level bureaucrats». En étudiant l'action publique "en train de se faire ", Lipsky montre que celle-ci ne se résume pas à la prise de décisions aux plus hauts niveaux des ministères, mais s'incarne chaque jour dans les interactions entre les usagers et les agents de base (p. 44). À partir des années 2000, les relations de service continuent à faire l'objet de nouvelles recherches, mais avec des travaux se réclamant cette fois d'une perspective plus "critique», inspirée par la sociologie de Pierre Bourdieu, comme ceux de Vincent Dubois ou de Yasmine Siblot (p. 50).

7 Le troisième chapitre porte sur les recherches s'intéressant à la dimension technique de l'activité. Dans ses recherches sur les collectifs ouvriers et leurs relations aux machines, Nicolas Dodier analyse la manière dont les objets techniques structurent les actions, mais peuvent aussi manifester une certaine autonomie vis-à-vis de leurs concepteurs en ouvrant vers des usages non anticipés et l'envie chez les travailleurs de développer des compétences nouvelles pour les maîtriser. Dans cette perspective, la technique n'est pas qu'un froid outil d'exercice de la domination des employeurs, il existe une «solidarité technique » qui lie les personnes et les objets dans une relation d'interdépendance. Elle est par ailleurs au centre de luttes auxquelles se livrent les membres d'un même métier dans des "arènes d'habilités techniques" (p.55). Ces propositions trouvent un écho dans les perspectives de la psychopathologie du travail ou de la clinique de l'activité développées à la même époque. Il ressort de ces approches une représentation du travail comme engagement du corps avec des techniques qui ne sont pas extérieures ou réductibles à une imposition par le haut, mais sont totalement imbriquées dans l'expérience individuelle et collective du travail (p.59). Les travaux d'Alexandra Bidet invitent à saisir l'activité dans un environnement, à partir de sa rythmicité et des formes d'engagement du corps et de soi plus généralement (p. 60). En enquêtant sur des milieux de travail fortement soumis aux automatismes, Alexandra Bidet dégage de ses observations tous « les efforts déployés par les travailleurs pour s'y retrouver » (p.60) et discriminer ce qui peut avoir le statut de "vrai boulot» (p. 62). D'autres recherches portent davantage sur le rôle de l'espace dans l'activité, la manière dont celui-ci est habité ou encore les mécanismes d'attention périphérique, de vigilance ou de coopération.

8 Dans un quatrième chapitre, Pascal Ughetto s'intéresse plus particulièrement aux sciences studies. Dans ce courant qui s'est développé à partir des années 1970, l'influence du pratice turn se retrouve dans les travaux qui se donnent pour objectif de reconstituer finement l'ensemble des activités indispensables pour que la science se fasse, au-delà de l'étude des grandes figures savantes. Cette démarche s'est notamment illustrée dans le célèbre ouvrage de Bruno Latour et Steve Woolgar «La vie de laboratoire» (1979). Dans cette perspective, la sociologie des sciences vise à rendre compte de la dimension collective de toute activité scientifique, en donnant à voir les différentes activités nécessaires à la production des résultats scientifiques, y compris les tâtonnements et les échecs, qui se révèlent parfois décisifs (p. 70). De même, elle tient à rendre visible la contribution de toute la chaîne des participants - humains et non humains - engagés 
dans la production d'un travail scientifique. Dans un souci de symétrie, ces approches s'intéressent aux "petites mains", aux "intermédiaires" autant qu'aux figures réputées scientifiques en défendant qu'il n'y a pas, d'un côté, la pure connaissance scientifique et, de l'autre, l'intendance. La science est ici vue comme une activité reposant sur une division du travail qui mérite une description minutieuse des pratiques, des espaces, des matériels de recherche, mais aussi des modèles théoriques utilisés (p. 74). Cette approche se retrouve dans des travaux anciens sur la médecine, comme ceux menés par Anselm Strauss et son équipe, ou ceux de Jean Peneff sur les chirurgiens et les urgences en France. En partant de la description de l'activité, ces travaux ont contribué à alimenter la connaissance des logiques professionnelles et organisationnelles.

9 Le cinquième chapitre s'intéresse aux relations interdisciplinaires de la sociologie de l'activité avec la linguistique, l'ergonomie, la clinique de l'activité ou encore la psychodynamique du travail. Pascal Ughetto rappelle notamment que les relations entre ergonomes et sociologues ont longtemps été "tendues", la place accordée à l'activité étant au cœur de leurs dissensions (p. 100). Alors que les ergonomes se voyaient reprocher par les sociologues d'adopter une focale trop réduite pour rendre compte de ce qui se joue dans les rapports sociaux de production ou, plus fondamentalement encore, de jouer le jeu du « réformisme » en cherchant à améliorer à la marge les conditions de travail, l'essor des recherches sociologiques sur le travail en train de se faire a permis des rapprochements partiels. La notion de «travail réel » (par opposition au « travail prescrit») développée par les ergonomes a par exemple été largement reprise par les sociologues pour mettre en exergue toute la complexité cognitive, y compris dans les tâches d'exécution qui reposent sur les travailleurs les moins qualifiés. Le rôle du CNAM et de figures comme Alain Winer ou Antoine Laville a aussi été déterminant dans le développement d'un dialogue interdisciplinaire et dans le dépassement de l'opposition entre sociologues critiques et compréhension fine de l'activité. La psychodynamique du travail développée par Christophe Dejours et son équipe a été un lieu où ont pu se rencontrer et dialoguer les descriptions minutieuses de l'activité et la critique des évolutions des organisations du travail et de leurs effets sur la santé (notamment mentale) des travailleurs. Dans style un peu différent, la clinique de l'activité développée par Yves Clot ou encore l'approche plus ancienne du médecin et psychologue du travail italien Ivar Oddone ont permis des collaborations régulières.

10 Le sixième et dernier chapitre interroge les possibilités de dialogue avec la sociologie du travail " classique ». Pascal Ughetto tient à rappeler que son intention n'est pas de durcir les contradictions ni de dramatiser une opposition entre une ancienne et nouvelle manière d'aborder le travail. Ce chapitre final cherche en particulier à montrer que là où il est reproché aux approches minutieuses de l'activité d'ignorer les rapports de pouvoir, «la réalité est plus compliquée» (p. 115). Pour la sociologie de l'activité telle que Pascal Ughetto la défend, «il s'agit de trouver une voie pour penser la prescription présente dans le travail et l'exécution du contrat de travail et de revisiter cette prescription à partir du prisme des pratiques» (p. 116). La relecture d'auteurs classiques comme Georges Friedmann tout comme la notion de «travail empêché » d'Yves Clot ou celle de "drame social » du travail de Hughes peuvent par exemple nourrir une sociologie de l'activité soucieuse de ne pas esquiver le débat sur le caractère hiérarchisé des organisations productives et les effets du taylorisme sur la santé des travailleurs. Finalement, Pascal Ughetto défend que si l'on veut bien faire 
jouer la diversité des ressources théoriques internes aux nouvelles sociologies du travail, l'articulation entre le micro et le macro dans l'analyse est accessible et permettrait à la fois de rendre compte des capacités des acteurs autant que des évolutions des organisations du travail.

11 En conclusion, la caractéristique cruciale de la sociologie de l'activité est de "voir le travailleur d'abord et avant tout à partir de son agency» (p. 131). Ce terme renvoie à la capacité d'action autonome des acteurs pour prendre leurs situations en main, affirmer le pouvoir sur leur travail et ainsi leur identité. Si cette perspective laisse au second plan l'analyse des stratégies déployées par les employeurs pour rationaliser l'organisation du travail et les conflits qui opposent les salariés à leurs supérieurs afin de mieux saisir comment les individus et les collectifs s'y prennent pour mener à bien leur activité, effectuer leur "vrai boulot " pour emprunter à la sociologue Alexandra Bidet, elle doit néanmoins viser à articuler les différents niveaux d'analyse entre « individu, collectif, organisation » (p. 132).

Riche et agréable à lire, cet ouvrage de synthèse relève finalement le défi de présenter un vaste ensemble de travaux de manière pédagogique tout en suscitant une réflexion sur différents enjeux auxquels les chercheurs font aujourd'hui face. Si les renouvellements théoriques au cœur de cet ouvrage sont à mettre en relation avec les évolutions du monde du travail, Pascal Ughetto souligne que certaines problématiques restent néanmoins peu traitées. La question des rapports de pouvoir dans la relation de travail ou encore celle du sens du travail demeurent souvent dans l'angle mort de ces «nouvelles sociologies». L'auteur propose ainsi des pistes comme celle de mieux articuler dans l'analyse les différentes dynamiques qui se jouent au travail à l'échelle de l'individu, du collectif, mais aussi de l'organisation. On pourrait ajouter d'autres pistes de réflexion: comment mener à bien de telles recherches dans un contexte où l'observation directe se confronte aux logiques de dématérialisation, de télétravail, de délocalisations ou encore de recul des organisations syndicales et des autres instances représentatives qui ont été, jusque dans la période des années 1990 et 2000, d'importantes pourvoyeuses d'accès aux terrains? Voici certaines des questions qui restent en suspens au terme de cet ouvrage. 